\title{
Statistical properties of low-frequency fluctuations during single-mode operation in distributed-feedback lasers: experiments and modeling
}

\author{
Tilmann Heil, Ingo Fischer, and Wolfgang Elsässer \\ Institute of Applied Physics, Darmstadt University of Technology, Schlossgartenstrasse 7, D-64289, Darmstadt, Germany \\ Josep Mulet \\ Instituto Mediterráneo de Estudios Avanzados, Consejo Superior de Investigaciones Científicas-Universitat de les Illes Balears, \\ E-07071 Palma de Mallorca, Spain
}

Claudio R. Mirasso

Departament de Física, Universitat de les Illes Balears, E-07071 Palma de Mallorca, Spain

Received May 12, 1999

\begin{abstract}
Extensive experimental and numerical investigations of feedback-induced instabilities in single-mode distributed-feedback lasers are presented that confirm the basic assumptions of the Lang-Kobayashi model. We give experimental evidence of the occurrence of low-frequency fluctuation (LFF), alternation between LFF and stable emission, and coherence collapse during single-mode operation of the laser. We have obtained quantitative agreement between modeling and experiment in long-time statistical investigations of the time intervals between subsequent LFF dropouts. In particular, we show that even the dependence of the dynamics on the injection current, which results in a scaling law, is quantitatively identical in modeling and experiment. (C) 1999 Optical Society of America
\end{abstract}

OCIS codes: $140.5960,140.1540$.

Semiconductor lasers show a rich variety of dynamic phenomena when they are subjected to moderate or even small amounts of delayed optical feedback. Aside from the fundamental interest in delay-induced instabilities, a profound understanding of these dynamic phenomena is indispensable for the stabilization of semiconductor laser emission (e.g., for photonic applications), for concepts to apply chaos control, and for synchronization of chaotic semiconductor lasers for applications in encoded communication. In this context, intensive research has been devoted to the understanding of the low-frequency fluctuation (LFF) phenomenon. ${ }^{1}$ LFF behavior is characterized by strong irregular fluctuations of the laser intensity that occur about the solitary laser threshold over wide ranges of optical feedback strength. Investigations of the LFF phenomenon have shown that a joint approach of experiments and numerical simulations is of decisive importance for possible advances. Usually, the numerical simulations are based on the Lang-Kobayashi (LK) rate-equation model, ${ }^{2}$ which assumes single-mode operation of the laser and neglects multiple reflections within the external cavity. However, in most experiments Fabry-Perot lasers, which exhibit multimode operation when they are subjected to delayed optical feedback, are used. Therefore, even though single-mode theory and multimode experiments are in qualitative agreement, this mismatch between theoretical assumptions and experimental conditions has attracted considerable attention. ${ }^{3,4}$ Here we aim to obtain an optimum match of experimental and theoretical conditions to provide an experimental system in which the results of numerical investigations can be realized straightforwardly in experiments. This is particularly important because chaos control $^{5}$ and synchronization ${ }^{6}$ have already been achieved in numerical simulations based on the LK model; stabilization has already been realized experimentally ${ }^{7}$ according to one of these numerical concepts. $^{8} \quad$ We use a single-mode distributed-feedback (DFB) laser with a large side-mode suppression ratio to fulfill the single-mode assumption of the LK model. Further, moderate amounts of optical feedback allow us to neglect multiple reflections. In our numerical simulations based on the LK model we use parameters that are estimated from the experiment. DFB-specific effects (e.g., interaction between a DFB grating and an external cavity) are neglected, as is well justified by the excellent agreement of our numerical and experimental results.

In the experiments we use a single-mode $1.55-\mu \mathrm{m}$ DFB semiconductor laser that is subjected to delayed optical feedback from an external cavity. Figure 1 depicts the optical spectra of the solitary laser and the laser with optical feedback. The spectra were recorded with a grating spectrometer with a resolution of $0.1 \mathrm{~nm}$. The significant linewidth broadening toward longer wavelength corresponds to predictions of the LK model. ${ }^{9}$ The laser persists in its single-mode operation with a very large side-mode suppression ratio of $40 \mathrm{~dB}$ during LFF operation. Figure 1 demonstrates that this single-mode operation holds even for fully developed coherence-collapsed operation of the laser. Thus we conclude that multimode operation is not required for the occurrence of LFF and feedbackinduced instabilities in semiconductor lasers.

The LK rate equations for the slowly varying amplitude of complex electric field $E$ and carrier number $N$ 


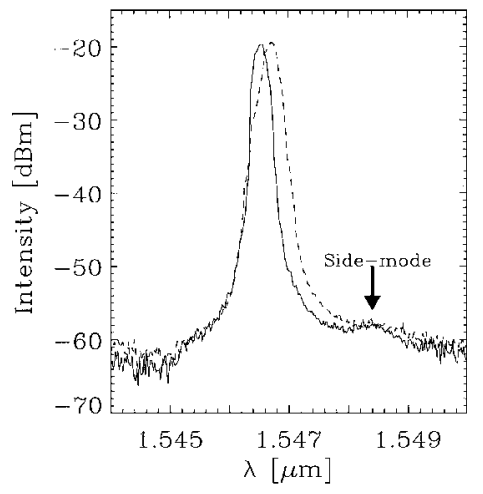

Fig. 1. Optical spectra of the DFB laser. Solid curve, the solitary laser; dashed curve, the laser with feedback under coherence-collapsed operation. $J=1.18 J_{\text {th }}$.

inside the cavity ${ }^{2}$ are

$$
\begin{aligned}
\frac{\mathrm{d} E(t)}{\mathrm{d} t}= & \frac{1}{2}(1+i \alpha)\left[G-\Gamma_{0}\right] E(t)+\gamma E(t-\tau) \\
& \times \exp \left(-i \omega_{0} \tau\right)+F_{E}(t), \\
\frac{\mathrm{d} N(t)}{\mathrm{d} t}= & p \frac{J_{\mathrm{th}}}{q}-\frac{N(t)}{T_{1}}-G P(t), \\
G(N, P)= & \frac{\xi\left(N-N_{t}\right)}{1+\epsilon P}, \quad P=|E|^{2} .
\end{aligned}
$$

The parameters used in our numerical simulations correspond to the following experimental conditions: delay time, $\tau=2.3 \mathrm{~ns}$; solitary threshold current $J_{\text {th }}=27 \mathrm{~mA}$, with $p=J / J_{\text {th }}$; and feedback rate, $\gamma=$ $14.35 \mathrm{~ns}^{-1}$, which reduces the threshold of the laser by $5.4 \%$. Differential gain $\xi=7 \times 10^{-6} \mathrm{~ns}^{-1}$; linewidth enhancement factor $\alpha=3.4$; number of carriers at transparency, $N_{t}=5.022 \times 10^{7}$; and carrier lifetime $T_{1}=0.45 \mathrm{~ns}$ are estimated from experimental measurements. The photon decay rate is $\Gamma_{0}=180 \mathrm{~ns}^{-1}$. $F_{E}$ is a Langevin noise force that describes random fluctuations caused by spontaneous emission. The random force has zero mean and is $\delta$ correlated in time, $\left\langle F_{E}(t) F_{E}{ }^{*}\left(t^{\prime}\right)\right\rangle=4 N \beta \delta\left(t-t^{\prime}\right)$, with $\beta \sim 10^{-7} \mathrm{~ns}^{-1}$, corresponding to a spontaneous-emission rate of $\sim 3 \times$ $10^{10} \mathrm{~s}^{-1}$. The equations are normalized such that $P=|E|^{2}$ is the number of photons inside the cavity. With the above parameters, the threshold current for the appearance of LFF has been determined to be $J_{\mathrm{th}}^{\mathrm{LFF}}=0.97 J_{\mathrm{th}}$, in agreement with the experimental value. For bias currents close above $J_{\mathrm{th}}^{\mathrm{LFF}}$ we observe, both experimentally and numerically, alternation between LFF's and stable operation on a single high-gain external-cavity mode (HGM). ${ }^{9,10}$ Figures 2(a) and 2(b) depict experimental and numerical time series, respectively, that are typical for this alternating behavior. Both time series have been obtained for $J=$ $0.98 J_{\text {th }}$. Within this regime the system jumps back and forth between LFF's and the HGM. This action is best demonstrated by the numerical simulations in $\eta-N$ space, where $\eta(t)=\phi(t)-\phi(t-\tau)$ corresponds to the phase difference of the electric field $E(t)$ in one round-trip time: The numerics in the inset of Fig. 2(b) confirm that the system reaches the HGM.
In numerical simulations without the noise term, the trajectory first undergoes several LFF dropouts, then reaches the HGM, and finally remains there. Only when the stochastic term is included in Eqs. (1)-(3) is the dynamic behavior depicted in Fig. 2(b) observed. This can be understood as follows: Once the trajectory has reached the stable emission state, ejecting the trajectory away from the HGM so the LFF can restart requires the presence of noise. In general, the stability of the external-cavity modes, i.e., their robustness against external perturbations or noise, strongly depends on the linewidth enhancement factor $\alpha$. Our numerical simulations demonstrate that the stability of the laser increases with decreasing $\alpha$, a result that corresponds to recent experimental findings with FabryPerot lasers. ${ }^{11}$

A joint approach of theory and experiment for the realization of chaos control and synchronization requires quantitative agreement of experiment and numerical simulations over long time intervals. Therefore we have performed long-time statistical investigations of the time intervals between subsequent LFF dropouts. Our extensive numerical simulations permit what we believe is the first quantitative comparison of experiment and theory on such long time scales. Experimentally, we have recorded 50 time series, each of $5-\mu \mathrm{s}$ length, covering several thousand power dropouts; numerically, we have considered at least $10^{4}$ dropout events. From these time series we obtained probability-density functions (PDF's) of the time intervals between subsequent power dropouts. Figure 3 depicts the corresponding experimental numerical PDF's for three injection currents that cover the whole LFF regime: Fig. 3(a), $0.98 J_{\text {th }}$; Fig. 3(b), $1.04 J_{\mathrm{th}}$; and Fig. 3(c), $1.08 J_{\mathrm{th}}$. The agreement of theory and experiment is excellent. All PDF's display similar features: an exponential decay for long times and a dead time for short times. The theoretically

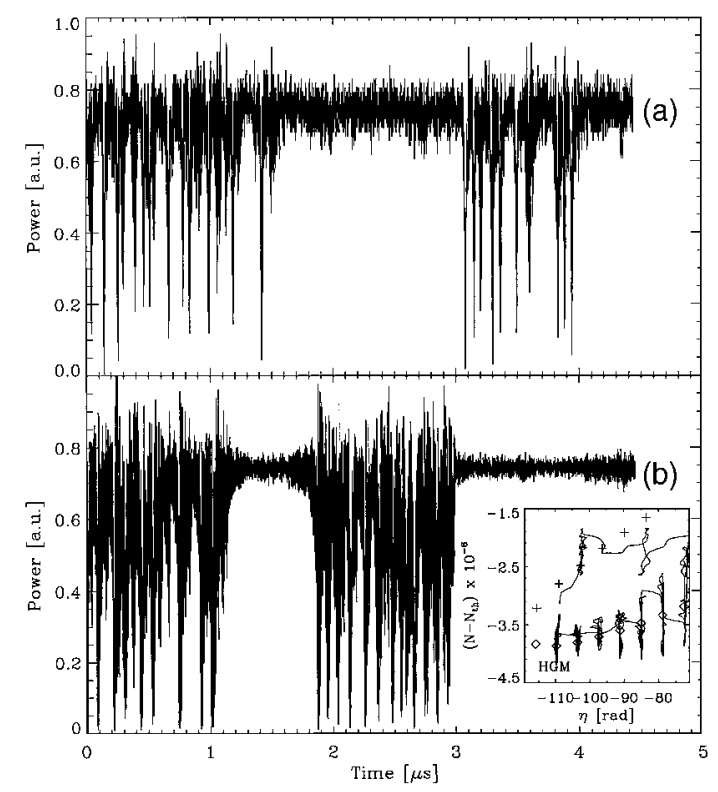

Fig. 2. Time traces showing alternation between LFF and stable operation: (a) experiment, (b) numerical simulations. The inset shows in $\eta-N$ space how the trajectory reaches the HGM. 


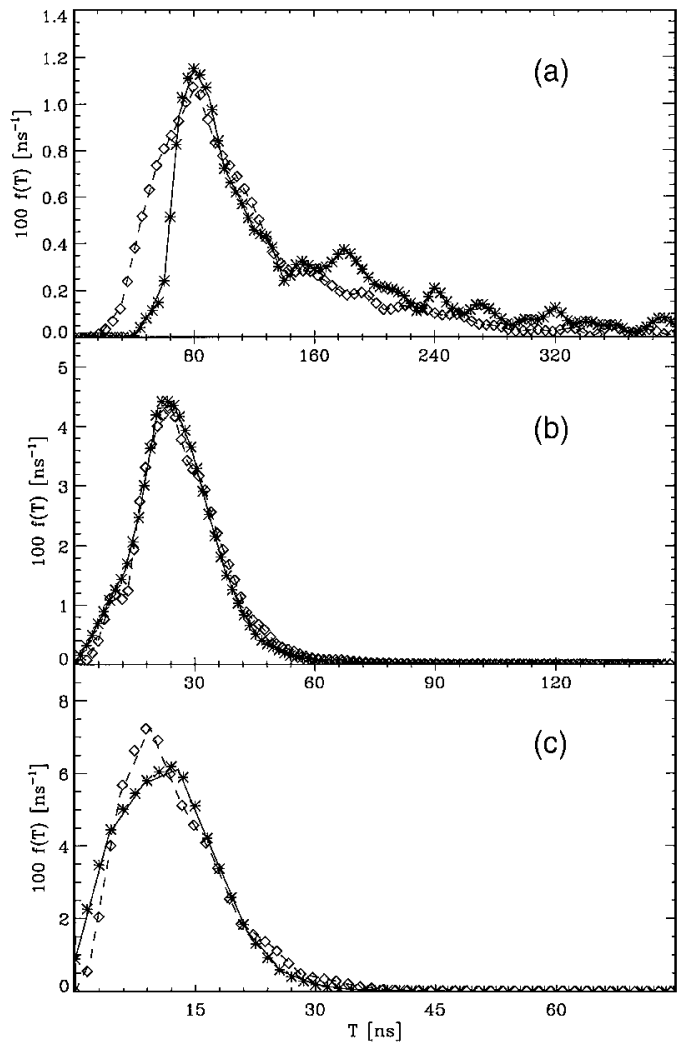

Fig. 3. PDF's $f(T)$ of time interval $T$ between subsequent LFF dropouts: (a) $J=0.98 J_{\text {th }}$, (b) $J=1.04 J_{\text {th }}$, (c) $J=$ $1.08 J_{\text {th }}$. Diamonds and dashed curve, experimental data; stars and solid curves, numerical simulations. Note the different scales of $f(T)$ and $T$ in (a) $-(\mathrm{c})$.

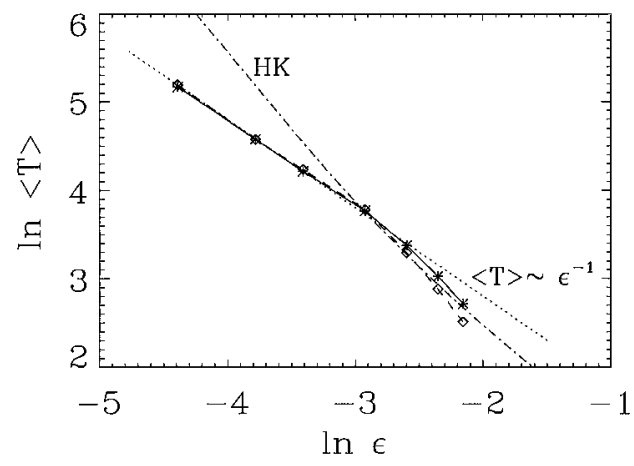

Fig. 4. Ln-ln plot of the average time interval between subsequent dropouts $\langle T\rangle$ versus normalized injection current $\epsilon=J / J_{\mathrm{th}}^{\mathrm{LFF}}-1$. Diamonds and dashed curve, experimental data; stars and solid curve, numerical simulations. The dotted curve corresponds to a linear fit $\langle T\rangle \sim \epsilon^{-1}$; the dashed-dotted curve, to results obtained from the Henry-Kazarinov ${ }^{12}$ model.

and experimentally obtained PDF's show an identical dependence on the injection current. In particular, the mean time interval between subsequent power dropouts $\langle T\rangle$ exhibits an interesting dependence on the injection current: Figure 4 depicts experimental and numerical data in a $\ln -\ln$ plot of $\langle T\rangle$ versus $\epsilon=J / J_{\mathrm{th}}^{\mathrm{LFF}}-1$, where the current is normalized with respect to the threshold current for the onset of the LFF. Both data sets demonstrate that, within the LFF regime, $\langle T\rangle$ scales as $\epsilon^{-1}$, whereas the fit based on the predictions by Henry and Kazarinov ${ }^{13}$ exhibits substantial deviations from the experimental and numerical results. The $\epsilon^{-1}$ scaling of $\langle T\rangle$ as well as the as the exponential decay and the dead time that are present in the PDF's have also been observed in experiments in which multimode Fabry-Perot lasers were used. ${ }^{13}$ This fact indicates that these properties are general ones that are present in the dynamics of semiconductor lasers, which consequently are independent of the number of optical modes involved in the laser emission. However, the mechanism that underlies the scaling is not yet fully understood. ${ }^{14}$

We have demonstrated coincidence between the requirements of the LK model and its realization by single-mode DFB lasers. In particular, we have given evidence for quantitatively identical parameter dependences of long-time LFF dropout statistics, including a characteristic scaling law for the mean time between the dropouts. Because chaos control ${ }^{5}$ and synchronization ${ }^{6}$ have been achieved numerically, the demonstrated quantitative agreement of the LK model and experiment strongly suggests directly using these techniques in experiments with single-mode DFB lasers.

We gratefully thank P. S. Spencer for providing the DFB lasers. This research was supported by the Deutscher Akademischer Ausfausddienst, Acción Integrada HA98-29, the Ministerio de Educación y Cultura of Spain, and the Deutsche Forschungsgemeinschaft Sonderforschungsbereich 185.

\section{References}

1. J. Mørk, B. Tromborg, and L. Christiansen, IEEE J. Quantum Electron. 24, 123 (1988).

2. R. Lang and K. Kobayashi, IEEE J. Quantum Electron. 16, 347 (1980).

3. G. Vaschenko, M. Giudici, J. J. Rocca, C. S. Menoni, J. R. Tredicce, and S. Balle, Phys. Rev. Lett. 81, 5536 (1998).

4. D. W. Sukow, T. Heil, I. Fischer, A. Gavrielides, A. Hohl, and W. Elsässer, Phys. Rev. A 60, 667 (1999).

5. C. Simmendinger and O. Hess, Phys. Lett. A 216, 97 (1996).

6. C. R. Mirasso, P. Colet, and P. Garcia-Fernandez, IEEE Photon. Technol. Lett. 8, 299 (1996).

7. A. Hohl and A. Gavrielides, Opt. Lett. 23, 1606 (1998).

8. J. Wieland, C. R. Mirasso, and D. Lenstra, Opt. Lett. 22, 469 (1997).

9. A. M. Levine, G. H. M. van Tartwijk, D. Lenstra, and T. Erneux, Phys. Rev. A 52, R3436 (1995).

10. T. Heil, I. Fischer, and W. Elsässer, Phys. Rev. A 58, R2672 (1998).

11. T. Heil, I. Fischer, and W. Elsässer, Phys. Rev. A 60, 634 (1999).

12. C. H. Henry and R. F. Kazarinov, IEEE J. Quantum Electron. 22, 294 (1986).

13. D. W. Sukow, J. R. Gardner, and D. J. Gauthier, Phys. Rev. A 52, R3370 (1997).

14. J. Mulet and C. R. Mirasso, Phys. Rev. E 59, 5400 (1999). 